\title{
Leveraging the Short-Term Memory of Hardware to Diagnose Production-Run Software Failures
}

\author{
Joy Arulraj Guoliang Jin Shan Lu \\ University of Wisconsin-Madison \\ $\{$ joy aliang shanlu @cs. wisc.edu $\}$
}

\begin{abstract}
Failures caused by software bugs are widespread in production runs, causing severe losses for end users. Unfortunately, diagnosing production-run failures is challenging. Existing work cannot satisfy privacy, run-time overhead, diagnosis capability, and diagnosis latency requirements all at once.

This paper designs a low overhead, low latency, privacy preserving production-run failure diagnosis system based on two observations. First, short-term memory of program execution is often sufficient for failure diagnosis, as many bugs have short propagation distances. Second, maintaining a short-term memory of execution is much cheaper than maintaining a record of the whole execution. Following these observations, we first identify an existing hardware unit, Last Branch Record (LBR), that records the last few taken branches to help diagnose sequential bugs. We then propose a simple hardware extension, Last Cache-coherence Record (LCR), to record the last few cache accesses with specified coherence states and hence help diagnose concurrency bugs. Finally, we design LBRA and LCRA to automatically locate failure root causes using LBR and LCR.

Our evaluation uses 31 real-world sequential and concurrency bug failures from 18 representative open-source software. The results show that with just 16 record entries, LBR and LCR enable our system to automatically locate the root causes for 27 out of 31 failures, with less than $3 \%$ run-time overhead. As our system does not rely on sampling, it also provides good diagnosis latency.
\end{abstract}

Categories and Subject Descriptors B.8.1 [Performance and Reliability]: Reliability, Testing, and Fault-Tolerance;

Permission to make digital or hard copies of all or part of this work for personal or classroom use is granted without fee provided that copies are not made or distributed for profit or commercial advantage and that copies bear this notice and the full citation on the first page. Copyrights for components of this work owned by others than ACM must be honored. Abstracting with credit is permitted. To copy otherwise, or republish, to post on servers or to redistribute to lists, requires prior specific permission and/or a fee. Request permissions from permissions@acm.org.

ASPLOS '14, March 1-5, 2014, Salt Lake City, Utah, USA.

Copyright @ 2014 ACM 978-1-4503-2305-5/14/03 .. \$15.00.

http://dx.doi.org/10.1145/2541940.2541973

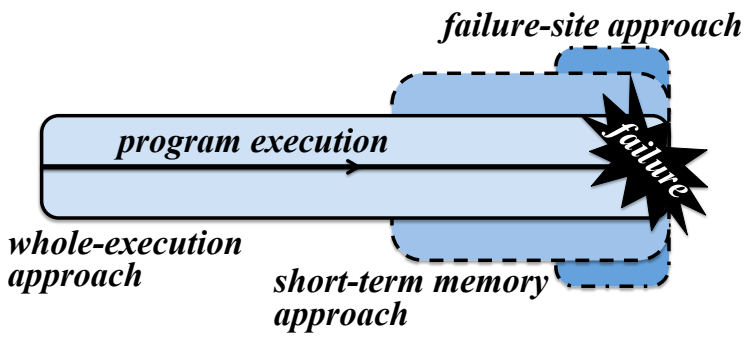

Figure 1: Approaches to diagnosing production-run failures (The rectangles illustrate program states directly collected by different approaches).

D.2.5 [Software Engineering]: Testing and Debugging; D.1.3 [Programming Techniques]: Concurrent Programming

Keywords failure diagnosis; production runs; concurrency bugs; hardware performance monitoring unit

\section{Introduction}

\subsection{Motivation}

Software bugs are widespread. Although effective bugdetection tools have been proposed, many software bugs inevitably slip into production runs. They have led to many severe production-run failures, causing huge financial loss [8, 17, 30, 36] and threatening people's lives [21]. Consequently, diagnosing failures that occur on production machines is a critical task.

Unfortunately, diagnosing production-run failures is challenging. Different from in-house bug detection and testing, production-run failure diagnosis has to preserve privacy and minimize run-time overhead, which often leads to sacrifices in diagnosis latency (i.e. how long it takes to diagnose a failure after its first occurrence) or diagnosis capability (i.e., what types of failures can be diagnosed).

Many tools have been proposed for production-run failure diagnosis. Since the occurrence of failures is difficult to predict, previous work either collects program states at the failure site, referred to as failure-site approach, or collects program states throughout the execution, referred to as whole-execution approach, as illustrated in Figure 1 These 
two approaches make different tradeoffs among privacy, runtime overhead, diagnosis capability, and diagnosis latency.

The failure-site approach incurs negligible run-time overhead, but has difficulty in satisfying the other requirements. Fundamentally, inferring run-time information using the program states at failure sites is not only tedious but also often impossible. Making things even worse, sending a lot of program states, such as the whole coredump, back to developers could severely hurt end users' privacy. Some of these problems are alleviated by recent work that uses static analysis to automate log-variable selection and backward inference [43-45]. However, static analysis is not a panacea. It cannot help when software fails at unexpected locations (e.g., during a segmentation fault). Its help is limited for failures caused by concurrency bugs. Its analysis time also increases with the number of logging sites.

The whole-execution approach can achieve better diagnosis capability than the failure-site approach due to its access to the whole execution information. On the down side, it can easily lead to huge run-time overhead. Many dynamic bug detectors are suitable for in-house testing but not for production-run use, because they either lead to huge overhead or require complicated non-existing hardware support. Recent work [2, 18, 22, 23] uses random sampling to address the overhead problem. However, random sampling leads to long diagnosis latency. For example, with the default 1 out of 100 sampling rate used by previous work [22, 23], a failure often needs to occur for about 100 times before the developers obtain information useful for failure diagnosis. This is especially a concern for software that is not deployed on millions of machines and bugs that manifest infrequently, such as concurrency bugs.

In summary, more tools are needed to support productionrun failure diagnosis.

\subsection{Contribution}

This paper presents a new approach to diagnosing a wide variety of production-run software failures with low run-time overhead and low diagnosis latency, while preserving end users' privacy. This new approach is based on the following two observations:

First, short-term memory is valuable and often sufficient for failure diagnosis. Previous empirical studies [12, 34, 48] have shown that most bugs have short propagation distances and hence have root causes located shortly before failures. Even for bugs with long propagation distances, information useful for failure diagnosis is generally not distributed evenly throughout the execution. Intuitively, information collected closer to a failure is more likely to be useful for diagnosis.

Second, short-term memory can be maintained with extremely low cost. In fact, existing hardware already maintains such short-term memory of software execution through facilities like Last Branch Record (LBR). With only 4-16 record entries maintained by $\mathrm{LBR}$, the hardware cost is low and the run-time overhead is negligible.

In short, maintaining a short-term memory of program execution can achieve a nice balance in the design space:

- Comparing with the failure-site approach, it has access to more run-time information and hence can achieve better diagnosis capability.

- Comparing with the whole-execution approach, it only keeps the most recent execution history and can achieve better performance without sacrificing diagnosis latency.

Following these observations, we propose a new production-run failure diagnosis approach that leverages the short-term memory of program execution maintained by hardware ${ }^{1}$. Several questions need to be answered to design such an approach.

First, what to remember in the short-term memory? A lot of hardware events occur during every machine cycle. We need to select hardware events that are most useful for diagnosing software failures.

Second, how large is short-term memory? Are 4-16 record entries, the settings in existing hardware LBR, sufficient for real-world failure diagnosis? Can this short-term memory provide information that cannot be inferred by the program states at the failure site? How often can this shortterm memory contain failure root-cause information? These questions have to be answered by thorough evaluation with real-world failures.

Third, how to use the short-term memory? We need to design a software system that accesses this hardware shortterm memory and integrates it into an automated failure diagnosis algorithm. The detailed implementation also needs to be careful not to pollute the precious short-term memory with irrelevant events, such as those from library or code used to access the short-term memory.

This paper answers the above questions and makes the following contributions:

- We propose a short-term memory approach to diagnosing production-run failures, with a good balance among privacy, run-time overhead, failure-diagnosis capability, and failure-diagnosis latency (illustrated in Figure 1 ).

- We identify and design two hardware short-term memory facilities to support production-run failure diagnosis. Specifically, we identify an existing hardware performance monitoring unit, LBR, to help diagnose sequential-bug failures, and propose a simple hardware extension, Last Cache-coherence Record (LCR), to help diagnose concurrency-bug failures. The details are in Section 4

\footnotetext{
${ }^{1}$ In this paper, we will refer to the hardware record of recent execution history as short-term memory. This short-term memory is composed of special machine registers, and has nothing to do with the main memory.
} 
- We design and implement two ways to use the hardware short-term memory for production-run failure diagnosis. The basic way, referred to as LBRLOG and LCRLOG, is to use LBR and LCR as a generic mechanism to enhance failure logging. It provides developers a straightforward and generic mechanism to obtain the execution history right before a failure, which often contains hints of failure root causes. The advanced way, referred to as LBRA and LCRA, uses a statistical model to automatically locate failure root causes from LBR/LCR records. The details are presented in Section 5 .

- A thorough evaluation based on 31 real-world failures from 18 open-source applications. Our evaluation based on 6945 failure-logging sites shows that more than $80 \%$ of LBR entries contain useful information that cannot be inferred by static control-flow analysis. LBRA can automatically locate branches that are closely related to failure root causes and bug patches for all the 20 evaluated sequential-bug failures, with less than $3 \%$ run-time overhead measured on commodity machines. In addition, LCRLOG and LCRA can help locate the root causes for 7 out of 11 tested concurrency-bug failures. Comparing with state-of-the-art systems that rely on sampling [2, 18, 22, 23], our failure-diagnosis system has tens to hundreds of times shorter diagnosis latency.

\section{Background}

\subsection{Hardware branch-tracing facilities}

There are two types of branch-tracing facilities in Intel processors. One is called Last Branch Record (LBR), which stores branch records in a circular ring of hardware registers. The other is called Branch Trace Store (BTS), which keeps branch records in cache or DRAM. BTS can store many more records than LBR. However, it incurs much larger overheads that is not suitable for production runs, ranging from $20 \%$ to $100 \%$ [31]. The following discussion will focus on LBR.

LBR is part of the hardware performance monitoring unit, originally designed for performance profiling. LBR branch recording uses special bus cycles on the system bus [14] and incurs negligible overhead. Its recording can be enabled and disabled through a special machine register, as shown in Table 1. Once enabled, LBR keeps recording newly retired branch instructions, with each new record evicting the oldest record. Each record contains the source and target addresses of a branch instruction. The total number of records in LBR varies in different microarchitectures, following an increasing trend over the years - it goes from 4 entries in Pentium 4 and Intel Xeon processors, to 8 in Pentium M processors, and to 16 in Nehalem processors [15]. All the experiments in this paper are conducted on an Intel Nehalem processor.

LBR can be configured to record different types of branch instructions, including conditional branches, unconditional jumps, calls, returns, and others, as shown in Table 1 .

\begin{aligned} & \hline IA32_DEBUGCTL ID: 0x1d9 \\ & \hline 0x801 Enable LBR \\ & 0x0 Disable LBR \\ & \hline LBR_SELECT ID: 0x1c8 \\ & \hline 0x1 *Filter branches occurring in ring 0 \\ & 0x2 Filter branches occurring in other levels \\ & 0x4 Filter conditional branches \\ & 0x8 *Filter near relative calls \\ & 0x10 *Filter near indirect calls \\ & 0x20 *Filter near returns \\ & 0x40 *Filter near unconditional indirect jumps \\ & 0x80 Filter near unconditional relative branches \\ & 0x100 *Filter far branches \\ & \hline\end{aligned}

Table 1: LBR related machine specific registers in Intel Nehalem (*: the masks used in this work).

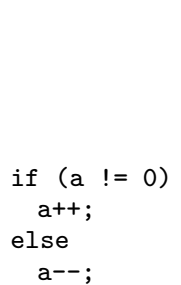

(a)

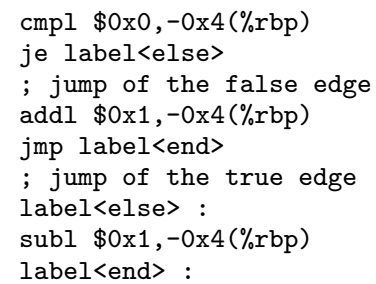

(b)
Figure 2: Conditional branches in source and machine code.

A subtle yet important issue in using LBR is that a conditional branch in source code does not simply map to a conditional branch in machine code. Figure 2 shows a simple example. The conditional branch in Figure 2 (a) is translated into one conditional jump instruction on Line 2 and one unconditional jump instruction on Line 5 in Figure 2 (b). The former will be taken when the original conditional branch is evaluated false, and the latter will be taken if the original branch is evaluated true.

Previous work proposes inserting harmless unconditional branches along the fall-through edges [40] to make the mapping between machine-code branches and source-code branches easier. We reuse this technique and skip the details.

In general, no matter the true edge or the false edge of a conditional branch in the source code is taken, some corresponding machine-level branch will get recorded in LBR. Developers will be able to locate the source-level branch and know its outcome based on the LBR record.

\subsection{Hardware performance counters}

Many modern processors equip each core with a few hardware performance-counter registers. These registers can be configured to monitor and count a wide variety of hardware performance events. Different from the branch tracing facil- 


\begin{tabular}{ll}
\hline LOAD (STORE) & Event Code : 0x40 $(0 \times 41)$ \\
\hline Unit mask & Description \\
\hline 0x01 & Observe I state prior to a cache access \\
0x02 & Observe S state prior to a cache access \\
0x04 & Observe E state prior to a cache access \\
0x08 & Observe M state prior to a cache access \\
\hline
\end{tabular}

Table 2: L1 data-cache cache-coherence events in Intel Nehalem (The event code and mask IDs are used to configure which type(s) of events to monitor).

ity discussed above, each such register only contains a count, corresponding to a specific performance event.

One set of events that can be monitored on many Intel processors, including Core 2 and Nehalem, is L1 datacache cache-coherence events [14]. As shown in Table 2 , once enabled, every L1 data cache access that encounters specified coherence state(s) will trigger a counter increment in the corresponding register. There are also configurations that allow various types of filtering, such as filtering out userlevel instructions or kernel-level instructions. The content of the register can be accessed through either polling or interrupts [2, 9]. We collectively refer to this set of L1 datacache cache-coherence events as cache-coherence events or coherence events in this paper. Note that, counting coherence events through hardware performance counters incurs no perceivable overhead on commodity machines [2, 14].

\section{Motivating Examples}

\subsection{Case 1: a sequential-bug failure}

Figure 3 shows a memory bug in sort utility from Coreutils. When a user tries to merge already-sorted files such that the output file is one of the input files, the program crashes inside the hash_lookup function.

This segmentation fault is caused by a buffer overflow in memmove within avoid_trashing_input (marked as $B$ in Figure 3). This buffer overflow corrupts files [i] .pid, which causes the control flow to deviate from the intended path at $C$. This eventually leads to a segmentation fault at $F$.

This buffer overflow is caused by the wrong whileloop condition at $A$. As we can see, the loop condition (i+num_merged $<$ nfiles) is intended to avoid buffer overflow. However, since the value of num merged is increased after this sanity check and before the access of files array, the buffer overflow occurs as long as this while-loop executes at least one iteration.

The control-flow uncertainty makes this failure very difficult to diagnose. First, the segmentation fault occurs in a function hash_lookup that has 9 different callers across 6 different files. Developers cannot even start their diagnosis without knowing the execution history. Second, even if the developers obtain the call-stack or even the core-dump from the end users' machines, they will likely ignore the

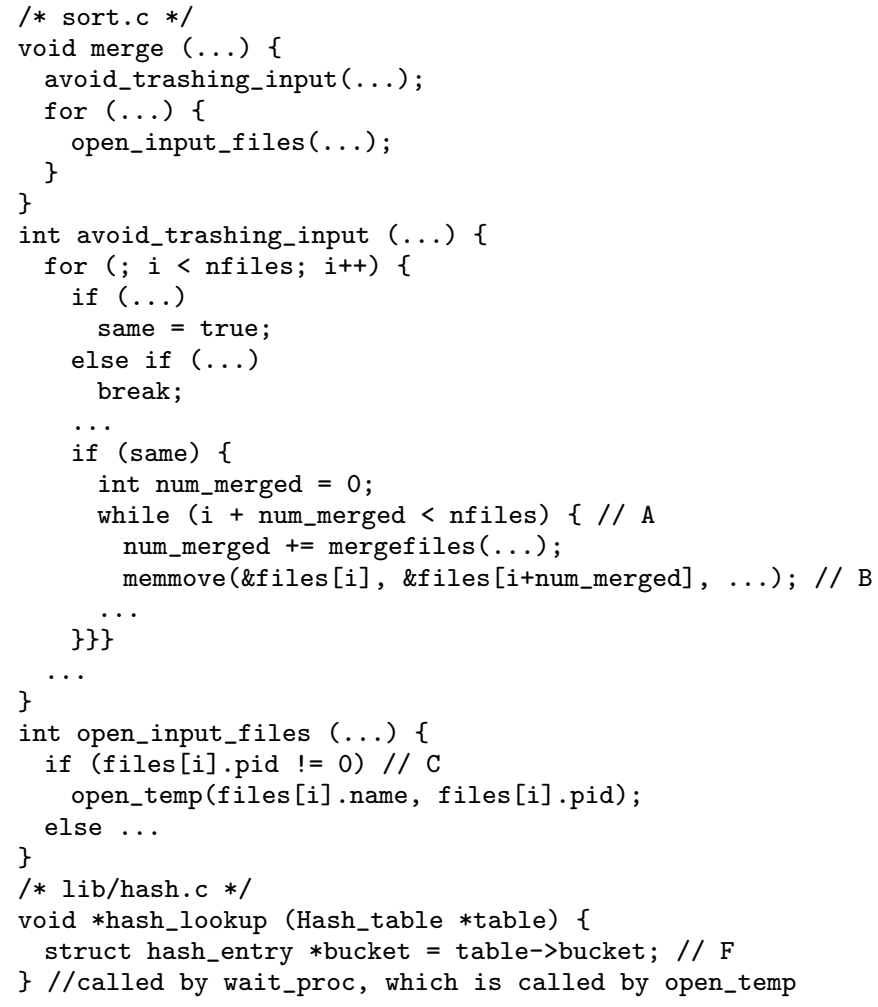

Figure 3: A sequential bug in sort utility in Coreutils-7.2.

avoid_trashing_input function, which is not on the call stack at the moment of failure. Third, even if developers pay attention to avoid_trashing_input, they do not know which basic blocks have executed, given the complicated control flow, not to mention discover the root cause at $A$.

In short, to effectively diagnose this failure, developers need to know the execution path leading to the failure. Otherwise, it is difficult to locate the root-cause code region and the root-cause branch. This path information often cannot be inferred by core-dumps, call-stacks, or log variables.

\subsection{Case 2: a concurrency-bug failure}

Figure 4 shows a concurrency bug in Mozilla JavaScript Engine. This bug is caused by unsynchronized accesses of the shared variable st->table. At runtime, the variable is initialized by InitState at $a_{1}$, and then checked at $a_{2}$. In most cases, this check will pass, as long as New has succeeded at $a_{1}$. Occasionally, st->table is set to NULL by another thread at $a_{3}$ right before the check is conducted. As a result, the software will fail with an "out of memory" message issued by ReportOutOfMemory.

Developers will encounter two major challenges in diagnosing this failure. First, control-flow uncertainties. The failure location in the source code is difficult to identify, because "out of memory" can be emitted by any one of the 55 locations where ReportOutOfMemory is invoked. Second, interleaving uncertainties. Even if the failure location 


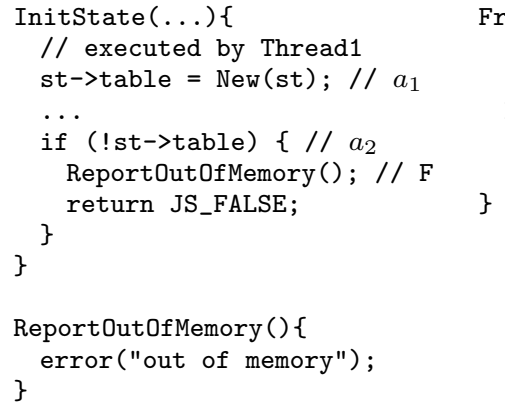

Figure 4: A concurrency bug in Mozilla JavaScript Engine.

is resolved, developers will probably mistakenly attribute the failure to memory-consumption problems in $a_{1}$, based on the control flow of InitState. Traditional log-enhancing techniques [44] provide little help here: developers cannot easily infer interleavings based on variable values logged at failurelogging site $F$, which is exactly why concurrency-bug failures are difficult to diagnose.

To successfully diagnose this failure, developers need at least two pieces of information: (1) "out of memory" is reported at $F ;(2)$ st->table is overwritten by another thread after the assignment at $a_{1}$ and before the checking at $a_{2}$. Both pieces can be collected from execution shortly before the failure. Unfortunately, existing production-run failurediagnosis techniques cannot deterministically provide these two pieces of information with low overhead.

\section{Maintaining Short-term Memory}

In this section, we identify and design hardware facilities that maintain short-term memory of program execution to support production-run failure diagnosis.

Our main task is to identify the right types of information to keep in the short-term memory. There is a wide variety of runtime information accessible to the hardware, such as the program counter of every executed instruction and the value stored in every register. We cannot record all these hardware events due to hardware cost and performance concerns. Therefore, we need to identify events that are most useful for failure diagnosis to keep in the short-term memory.

\subsection{LBR for sequential-bug failure diagnosis}

The outcome of conditional branches in software is among the most useful information for failure diagnosis. It can address the control-flow uncertainties discussed in Section 3 , explicitly presenting the execution path leading to the failure. In addition, previous work has shown that many sequential-bug failures are exactly caused by control-flow problems [23, 35].

Fortunately, the hardware facility that maintains the short-term memory of this information already exists in the form of Last Branch Record (LBR). There are different types of branches that could be recorded in LBR. Our system con- figures LBR to record three types of branches that can help resolve the outcomes of conditional branches in user-level programs, as shown in Table 1 .

\subsection{LCR for concurrency-bug failure diagnosis}

\subsubsection{LCR design}

Previous work [2] has found coherence events maintained by existing hardware performance counters, which are discussed in Section 2.2, useful in diagnosing concurrency-bug failures. Inspired by that, we propose a hardware extension that maintains the short-term memory of coherence events. We call it Last Cache-coherence Record, short as LCR.

Assuming a MESI cache-coherence protocol, LCR includes the following components on chip:

1. A special hardware register that configures which type of coherence events to record. The supported events are $e x$ actly those that can already be counted by existing hardware performance counters - load or store instructions that observe certain cache-coherence states right before the cache access, as discussed in Section 2.2. Detailed configuration options follow those that are provided by existing hardware for performance counter registers, as also discussed in Section 2.2 For example, the cachecoherence state can be any combination of modified state, exclusive state, shared state, and invalid state; this register can be configured to filter out kernel-level instructions or user-level instructions from LCR.

2. $K$ pairs of special hardware registers per core that record the latest $K \mathrm{LCR}$ events. Each pair records the instruction counter and the specific cache-coherence state observed by that instruction ${ }^{2}$ By default, we set $K$ to be 16 , resembling the setting of LBR on Nehalem processors.

3. Extra circuits that keep updating LCR on each core. After the retirement of L1 data-cache access instructions, the program counter of the instruction and the cache-coherence state observed by this instruction will be recorded in LCR, if the state matches the configuration.

We expect LCR to be a simple extension on machines that already support hardware cache-coherence performance events and LBR, such as Intel machines discussed in Section 2.2 LCR essentially requires extending these machines from being able to count cache-coherence events to being able to record while counting, just like building LBR in machines that can count branch-taken events.

It is difficult to accurately estimate the overhead incurred by LCR without building it in real hardware. We expect the overhead to be low for two reasons. (1) Counting cachecoherence events incurs no perceivable overhead on commodity machines [2, 14]. (2) Using LBR incurs negligible overhead on commodity machines, as shown in Section 8

\footnotetext{
${ }^{2}$ Memory addresses are not recorded
} 


\begin{tabular}{|c|c|c|c|c|}
\hline Bug Type & FPE & Does FPE exist in failure thread? & \multicolumn{2}{|l|}{ Example } \\
\hline RWR Atomicity Violation & Invalid Read & Almost Always & $\begin{array}{l}\text { /*Thread } 1 * / \\
\text { if(ptr) } / / a_{1} \\
\quad \text { puts(ptr);//a }, \mathrm{F}\end{array}$ & $\begin{array}{l}/ * \text { Thread } 2 * / \\
\text { ptr=NULL; //a } 3\end{array}$ \\
\hline RWW Atomicity Violation & Invalid Write & Almost Always & $\begin{array}{l}/ * \text { Thread } 1 * / \\
\operatorname{tmp}=\mathrm{cnt}+\text { deposit } 1 ; / / a_{1} \\
\mathrm{cnt}=\mathrm{tmp} ; / / / a_{2} \\
\text { printf("Balance=\%d", cnt); //F }\end{array}$ & $\begin{array}{l}/ * \text { Thread } 2 * / \\
\mathrm{tmp}=\mathrm{cnt}+\text { deposit } 2 \\
\mathrm{cnt}=\mathrm{tmp} ; / / a_{3}\end{array}$ \\
\hline WRW Atomicity Violation & Invalid Read & Sometimes & $\begin{array}{l}/ * \text { Thread } 1 * / \\
\log =\text { CLOSE; } / / a_{1} \\
\log =\text { OPEN; } / / a_{2}\end{array}$ & $\begin{array}{l}\text { / } * \text { Thread } 2 * / \\
\text { if }(\log !=\text { OPEN }) / / a_{3} \\
\{/ / \text { output failure }\} / / \mathrm{F}\end{array}$ \\
\hline $\begin{array}{l}\text { Read-too-early Order Violation } \\
\text { Read-too-late Order Violation }\end{array}$ & $\begin{array}{l}\text { Exclusive Read } \\
\text { Invalid Read }\end{array}$ & $\begin{array}{l}\text { Often } \\
\text { Often }\end{array}$ & $\begin{array}{l}\text { Figure } 5 \\
\text { Figure } 6\end{array}$ & \\
\hline
\end{tabular}

Table 3: The failure predicting events (FPE) of concurrency bugs ( Invalid and Exclusive refer to the cache-coherence state observed by a load or store right before it accesses L1 data-cache; F denotes the location of failure.)

Since our LCR design is built upon existing hardware cache-coherence performance events, it will share some constraints of existing hardware performance counters. For example, the LCRs on different cores are separately maintained and accessed, just like how existing performance counters work. When we profile LCR from a particular thread in a multi-threaded program, only the LCR maintained by the core that is currently running this thread will be accessed. Extending LCR to access multiple cores' information simultaneously would require non-trivial change to existing hardware. As another example, on SMT machines, multiple hardware threads in one core currently share one LBR. We expect a similar design for LCR. This will shorten the execution history recorded for each thread.

\subsubsection{How useful is LCR?}

Is LCR helpful in diagnosing concurrency-bug failures? To some extent, the answer is always "yes", because LCR can help developers understand the thread interaction right before failures.

Can LCR directly point out the failure root cause? It depends on whether LCR contains a coherence event that is failure predicting and is from the failure thread. An event is considered failure predicting, if it mostly occurs during failure runs and is related to the failure root cause [2, 18, [23]. A failure thread is the thread where the failure first occurs, such as the thread that encounters a segmentation fault, violates an assertion, and so on. Coherence events that occur outside the failure thread cannot be obtained when we access LCR at the failure site.

The above question is partly answered by our previous work [2], which shows that failure predicting coherence events exist for all common types of concurrency bugs. The remaining question is whether such events occur in failure threads or not. In the following, we discuss this issue for two most common types of concurrency bugs: single-variable atomicity violations and order violations [25].

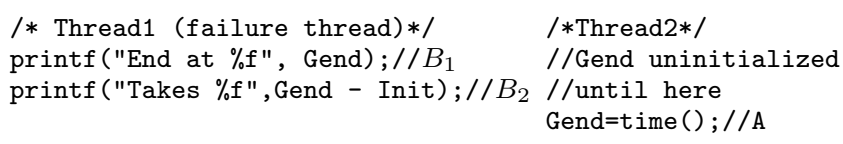

Figure 5: A read-too-early order violation in FFT. Thread 2 should initialize Gend before thread 1 accesses it.

Single-variable atomicity violations occur when two consecutive memory accesses from one thread, denoted as $a_{1}$ and $a_{2}$, are unserializably interleaved by an access, denoted as $a_{3}$, from another thread. All four types of single-variable atomicity violations are demonstrated in Table 3 . Our previous work [2] discovered that failure predicting events exist at $a_{2}$ for all these atomicity violations 3 Therefore, we will focus on discussing whether $a_{2}$ is in the failure thread below.

$a_{2}$ almost always exists in the failure thread for RWR and WWR atomicity violations, as the incorrect value read by $a_{2}$ will soon lead to failure in the same thread as $a_{2}$, such as a segmentation fault inside puts (the RWR example in Table 3) and the out-of-memory failure in Figure 4 . Here, we leverage observations from previous empirical studies [47, 48], which show that concurrency-bug failures almost always occur in the thread that first reads an incorrect value from a shared variable.

$a_{2}$ often exists in the failure thread for RWW atomicity violations - after $a_{2}$ writes an incorrect value, the thread performing $a_{2}$ will very likely use this incorrect value which will lead to failure, as shown in the example in Table 3 .

For WRW atomicity violations, failures usually occur in the thread containing $a_{3}$, instead of $a_{2}$, as shown in Table 3 Unfortunately, $a_{3}$ is not always a failure predicting event, unless it is preceded by another access to the same variable.

\footnotetext{
${ }^{3}$ The rationale is that $a_{2}$ would encounter different cache-coherence states during failure runs and success runs, due to the impact of $a_{3}$. For example, the invalid state of st->table encountered by if (!st->table) in Figure 4 is related to the failure root cause and can predict the failure.
} 


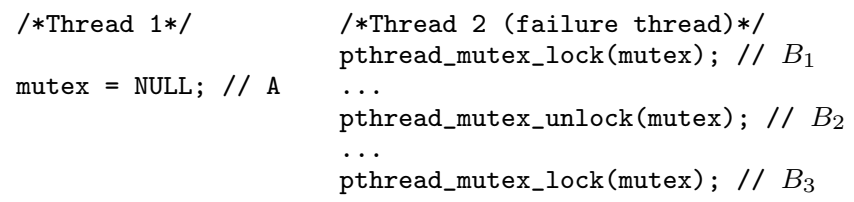

Figure 6: A read-too-late order violation in PBZIP2. Thread 2 should use mutex before thread 1 destroys it.

Order violations occur when the expected order between two operations from two threads is flipped. The two most common types of order violations occur either when a read instruction executes too early and hence accesses an uninitialized value (Figure 5) or when a read instruction executes too late and hence accesses a stale value (Figure 6). Previous work [2] has shown that the coherence event at the read instruction is often a failure predicting event. For the example shown in Figure 5, $B_{2}$ would encounter an exclusive state only during failure runs, when Gend is uninitialized. For the example shown in Figure 6, $B_{3}$ rarely encounters an invalid state during success runs, but always encounters an invalid state during failure runs when $B_{3}$ executes after the NULLassignment from another thread.

The above failure predicting events do exist in failure threads, as the incorrect values returned by the read instructions quickly lead to failures, such as wrong outputs (Figure 5) and crashes (Figure 6).

In summary, LCR has a good chance of directly pointing out the root cause of almost all common types of concurrency-bug failures. Even if the root cause cannot be directly pointed out, the thread interaction information provided by LCR is still helpful.

LCR configuration For extensibility and generality, we have designed LCR to record a wide variety of coherence events. Following the discussion in Table 3 , the following two configurations are most useful for diagnosing user-level concurrency-bug failures.

The first configuration records invalid loads, invalid stores, and exclusive loads. These events cover all the different types of failure-predicting events for common concurrency bugs, as shown by Table 3 However, this configuration may waste the LCR space with stack accesses that are often exclusive loads.

A more space-saving configuration is to replace exclusive loads with shared loads, using the latter to replace the former in diagnosing read-too-early order violations. For instance, consider the bug shown in Figure 5. During success runs, $B_{2}$ will always encounter a shared state. Therefore, failures are highly correlated with $B_{2}$ not encountering a shared state. This configuration is more LCR space-saving than the first one, but it may not be as straightforward as the first configuration for developers to reason about.

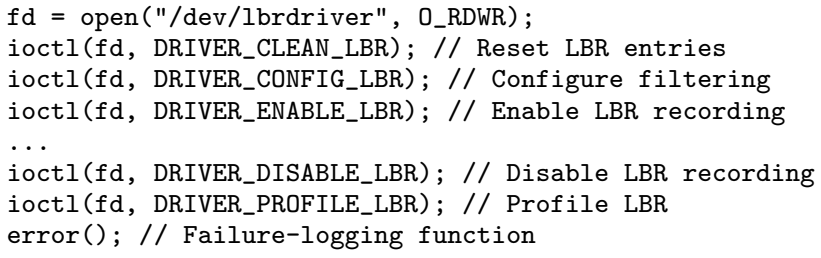

Figure 7: Interface exported by our LBR kernel module.

\subsection{Implementation details}

Accessing LBR This includes three steps. First, we configure which types of branches to record through the special register LBR_SELECT, as shown in Table 1 Second, we enable LBR through the IA32_DEBUGCTL register. Finally, the records in LBR can be accessed through registers BRANCH_0_FROM_IP through BRANCH_16_FROM_IP, where BRANCH_n_FROM_IP is the linear address of the $n^{\text {th }}$ branch instruction. Since the above registers cannot be accessed at user level, we implemented a Linux kernel module to support accesses from user level, mainly using kernel wrapper functions that execute rdmsr and wrmsr assembly instructions. The interface is shown in Figure 7

Minimizing LBR pollution As mentioned earlier, LBR has a limited capacity - 16 branch entries in Nehalem processors. To minimize the LBR pollution by branches that are irrelevant to user-level software failures, we use the following methods.

First, as discussed in Section 4.1. we configure LBR to filter out kernel level branches.

Second, we always disable LBR right before we read LBR. Our LBR-disabling code does not contain any userlevel branches. Consequently, LBR will not be polluted by code executed to access it.

Finally, we remove pollution from common library functions through a collection of wrapper functions. Each wrapper function disables LBR on entry, invokes the original library function, and finally enables LBR on exit. We will refer to this method as LBR toggling. We wrote wrappers for glibc functions and application-specific error-reporting functions. For example, for MySQL, we wrote wrappers for three MySQL-specific functions: _db_doprnt, my_error, and sql_print_error. Each wrapper function contains fewer than 15 lines of code and follows the same routine.

LCR simulation We expect that LCR will be added into the hardware performance monitoring unit in the future and will be accessed in a similar way as we access LBR. We expect that the pollution issue will be similarly solved by toggling around common library functions, filtering out kernellevel instructions, and disabling LCR before profiling.

We implement a LCR simulator using PIN binaryinstrumentation infrastructure [27]. Our LCR simulator includes two parts. The first part is a simulated L1-cache with MESI coherence protocol, implemented by instrumenting 
every memory instruction in the user program and libraries. The second part simulates LCR configuration, disable, enable, and profiling functions. We implement LCR as a perthread circular buffer with a configurable size. Once enabled, every thread's circular buffer gets filled by program counters and coherence states of instructions that are executed by the specific thread and satisfy the LCR configuration Once disabled, every thread's circular buffer is frozen. When a thread executes the profiling function, that thread's circular buffer content is retrieved. Finally, we simulate the pollution effect of these four functions by adding dummy entries into the corresponding circular buffer. Specifically, two user-level exclusive reads will be introduced by the ioctl call that enables LCR; two user-level exclusive reads and one user-level shared read will be introduced by the ioctl call that disables LCR.

Our simulation does not simulate OS events, such as exceptions and context switches. However, we believe it is accurate enough to provide a solid evaluation of LCR.

\section{Using Short-term Memory}

\subsection{Log enhancement}

The basic way of using LBR and LCR is to enhance failure logging, which we will refer to as LBRLOG and LCRLOG respectively. Developers can use the LBR/LCR record collected at a failure site to reconstruct the control flow and interleaving right before the failure. They may also find failure predicting events from the LBR/LCR record.

To ease the adoption and evaluation of LBRLOG and LCRLOG, we implemented a source-to-source code transformer to automatically enhance a program's failure logging. The transformation includes several steps:

(1) Changing the program compilation configuration to use our wrappers for common library functions (Section 4.3 .

(2) Inserting LBR/LCR configuration and enabling code at the entry of main function, as shown in Figure 7 .

(3) Inserting LBR/LCR profiling code right before every existing failure-logging function in the program, as shown in Figure 7. Currently, our implementation takes a developer configurable list of application-specific failure-logging functions, such as ap_log_error in Httpd and error in GNU core utilities software.

(4) Registering a custom segmentation-fault signal handler to profile LBR/LCR.

\subsection{Automatic failure diagnosis}

A more sophisticated way of using LBR/LCR is to automatically locate failure predicting events based on the LBR/LCR content collected from production runs. To achieve this goal, we have designed LBRA and LCRA.

LBRA/LCRA follows the existing statistical fault localization approach [22, 23]. It compares information collected from failure runs (i.e., failure-run profiles) with information

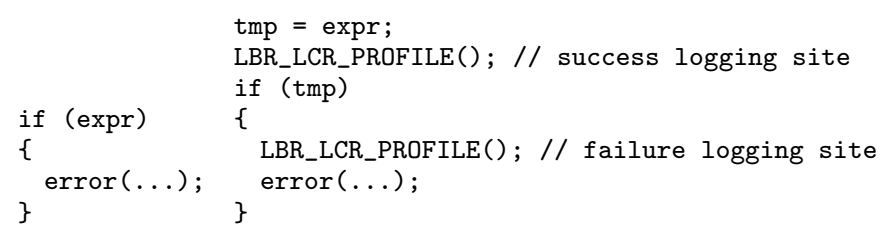

(a) Original code

(b) Transformed code

Figure 8: The logging sites for success and failure profiles.

collected from success runs (i.e., success-run profiles) to figure out events that are most correlated with failure runs. We will go through the design of these three components below:

1. What is the failure-run profile and how to collect it?

2. What is the success-run profile and how to collect it?

3. How to make the comparison?

Failure-run profile A good failure-run profile should have a high chance to contain failure-predicting events. In our system, we use the content of LBR and LCR collected by LBRLOG and LCRLOG as the failure-run profile. Clearly, the profile would contain exactly one LBR/LCR record for each run where a fail-stop failure occurs. For example, when the sort bug shown in Figure 3 manifests, a segmentation fault would occur at location $F$. The LBR/LCR record collected inside that segmentation fault handler would become the failure run profile.

Success-run profile LBRLOG and LCRLOG do not profile LBR and LCR at all during success runs. Therefore, we need to design success-run profiling separately from LBRLOG and LCRLOG.

Intuitively, we expect success-run profiles to contain LBR/LCR record collected nearby the failure sites, so that success-run profiles and failure-run profiles are comparable.

Guided by this intuition, we define the following program locations as success logging sites: (1) If a segmentation fault can be triggered by instruction $i$, the program location right after $i$ is a success logging site; (2) If a failure logging function is located at $F$, a success logging site is right before where the program jumps to the basic block containing $F$, as shown in Figure 8

Collecting LBR/LCR record at the success logging sites defined above will naturally provide success-run profiles that are comparable with failure-run profiles. In addition, it also naturally excludes irrelevant success runs from the failure diagnosis process - LBR/LCR will not be profiled during runs that do not execute the code around the failure site.

We have implemented two schemes to collect LBR/LCR at the success logging sites. The proactive scheme inserts LBR/LCR profiling code at every success logging site corresponding to every failure logging site before software release. The reactive scheme waits until a failure occurs at a particular location $F$, and then inserts LBR/LCR-profiling 
code at the success logging site corresponding to $F$. This change can be conducted either on the end users' machines through dynamic binary transformation [5] or at the development site. In the latter case, the changes will be propagated to the end users in the form of code patches.

These two schemes each have their own strengths. The proactive scheme does not require code redistribution, but incurs higher run-time overhead due to more frequent LBR/LCR profiling. In addition, it cannot help diagnose failures that manifest at unexpected locations, which is always the case for segmentation faults. The reactive scheme has better performance. However, it needs software updates to collect success-run profiles, which may take time. Of course, since failure runs are much rarer than success runs, delays in collecting success-run profiles rarely lead to longer diagnosis latency.

How to compare? Each success/failure run profile is a set of events recorded in LBR and LCR. We want to identify the event whose occurrence can best predict the failure.

Similar to previous work on statistical debugging [2, 23], we identify the best failure-predicting event based on the expected prediction precision and recall of the events. Specifically, in our context, prediction precision measures how many runs indeed fail among those that are predicted to fail by the event. It can be calculated by $\frac{|F \& e|}{|e|}$, where $|e|$ denotes the number of runs where $e$ is recorded in the profile and $|F \& e|$ denotes the number of failure runs that contain $e$ in their profiles. Prediction recall measures how many runs are predicted to fail by the event among those that indeed fail. It can be calculated by $\frac{|F \& e|}{|F|}$, where $|F|$ denotes the number of failure runs. We rank all events based on the harmonic mean of the expected prediction precision and recall, and identify the highest ranked event as the best failure-predicting event.

\subsection{Discussion}

Failure sites In our current implementation, LBRLOG and LCRLOG treat existing failure-logging functions and segmentation-fault handler as failure sites. Consequently, if software fails by silently corrupting data, LBR and LCR will not be collected in a timely manner and hence may not help the diagnosis. Fortunately, the problem of identifying the right places to insert log functions has been largely addressed by recent work [45].

Multiple failures It is very natural for large software to encounter failures caused by different bugs during production runs. The existence of multiple failures will not affect our system. From each failure-run profile, we can identify the location of the failure site and hence separately handle failures that occur at different program locations. Very rarely, different root causes may lead to failures at the same location, such as the example shown in Figure 4 In this case, we will see that even the best failure predicting event does not appear in every failure run. Fortunately, this rarely affects the relative ranking among events, and our system can still identify the best failure predicting events.

Log enhancement Traditional failure logging either dumps core images or call stacks, or records the values of selected program variables. LBR/LCR has its unique advantages and can well complement these traditional approaches.

In terms of preserving privacy, LBR/LCR is among the best, because it does not directly collect any variable values.

In terms of failure diagnosis capability, LCR provides interleaving related information that is difficult to obtain from traditional approaches. LBR can resolve uncertain control flows that cannot be inferred by traditional approaches. Note that, even the recently proposed LogEnhancer [44] can have difficulty in resolving control flows in sibling functions (i.e. functions that are not in the call-stack at the failure site). Of course, LBR and LCR content is limited to the execution shortly before the failure. In addition, coredumps and logging variables can provide concrete variable value information that may be unavailable from LBR/LCR.

In terms of logging latency, logging LBR/LCR is much faster than dumping cores and logging call-stacks. In our evaluation, logging LBR/LCR takes less than $20 \mu \mathrm{s}$; dumping core can easily take more than $200 \mathrm{~ms}$; and recording the call stack takes about $200 \mu \mathrm{s}$. If developers want to conduct logging at locations beyond failure sites, logging LBR/LCR is more suitable than dumping cores or call-stacks.

In terms of run-time performance, LBR/LCR profiling incurs negligible overhead if toggling is disabled. Enabling toggling around library functions could cause perceivable overhead for applications that frequently invoke library functions, which will be evaluated in Section 7

Finally, logging LBR/LCR is more generic than logging selected variables. At different program locations, different variables need to be selected to represent the most important program states for failure diagnosis. When software fails at an unexpected location, such as during a segmentation fault, variable logging usually cannot be applied.

Automated failure diagnosis The cooperative bug isolation (CBI) approach, including CBI [22, 23], CCI [18], and PBI [2], are the state of the art production-run failure diagnosis techniques. This approach first evaluates certain predicates, such as whether a branch is taken, at randomly sampled locations. It then performs statistical analysis on the data collected from many success and failure runs to identify predicates that strongly correlate with failures. These predicates are referred to as failure predictors. To conduct random sampling, CBI and CCI use source-code instrumentation, while PBI leverages hardware performance counters.

LBRA/LCRA has much shorter diagnosis latency than the CBI approach. Suppose $e$ needs to occur in a couple of failure-run profiles to be identified as a high-confidence failure predictor. To identify $e$, LBRA/LCRA needs a failure to occur for a couple of times. The CBI approach needs a failure to occur for hundreds of times under their default 
sampling rate (1 out of 100). This difference, hundreds of failure occurrences, could mean a long time in practice, because bugs that slip into fields often manifest rarely.

In terms of run-time performance, LBRA/LCRA is better than $\mathrm{CBI} / \mathrm{CCI}$ and is comparable with PBI. The advantage of LBRA/LCRA mainly comes from two sources. First, LBRA/LCRA collects failure-run profile only at one location, the failure-logging site. Instead, the CBI approach periodically evaluates predicates throughout the execution. Second, CBI and CCI pay extra cost to enable their random sampling. This overhead is often more than $30 \%$ for CPUintensive applications for CBI [3] and more than $800 \%$ for CCI [2].

In terms of diagnosis capability, LBRA/LCRA is comparable with PBI and CCI when failures have reasonably short propagation distances, which is true for most failures [12, 29, 34, 48]. CBI is better than LBR-tools for sequentialbug failures whose root causes are not related to branches.

Finally, LBRA and LCRA have a much smaller impact on the executable-file size than CBI and CCI, leaving a much smaller footprint on cache and memory.

Limitations The accuracy of our LBR/LCR based tools could be slightly affected by certain hardware issues. Hardware tracks the cache-coherence states at cache-line granularity, instead of variable granularity. This could lead to false sharing problems. Invalid cache states could be caused by both cache eviction and remote write accesses. This could cause one coherence event to appear in both success runs and failure runs. Of course, since the ranking model discussed in Section 5.2 naturally filters out random noises, we expect the diagnosis results to be rarely affected by these issues.

\section{Methodology}

We conduct all the experiments on an Intel Core 17 machine with 4 physical cores running Linux 3.5 kernel. We separately evaluate LBR and LCR related tools. LBR-related experiments are conducted directly on the real machine; LCRrelated experiments are conducted on our PIN-based simulator.

We evaluate the failure-diagnosis capability and runtime performance of LBRLOG and LBRA using 20 real-world sequential-bug failures. We include in our benchmarks all the 10 failures from LogEnhancer [44] that we can reproduce. We also randomly pick 5 failures from 13 reproducible crash failures from Errlog [45]. Finally, since the above failures are all from $\mathrm{C}$ applications, we randomly selected 5 reproducible bugs from the bug database of open-source $\mathrm{C}++$ applications Cppcheck and PBZIP.

To measure performance, we use workloads designed by the software developers that represent the common scenarios in production runs and do not lead to failures. The performance overheads reported are the mean of 10 measurements. To evaluate failure-diagnosis capability, we use the bug triggering inputs used by LogEnhancer, Errlog, and the original bug reports. For all these benchmarks, we conduct a head-to-head quantitative comparison between LBRA and CBI. Further, we evaluate how LBRLOG can help resolve control-flow uncertainties using all the 6945 logging points in 13 open-source applications. Detailed information about these benchmarks along with the main logging functions instrumented by LBRLOG is shown in Tables 4 and 5 .

We evaluate the failure-diagnosis capability of our LCR proposal using all the 11 real-world concurrency-bug failures used in PBI [2] and CCI [18], following their experiment settings. Our LCR simulator simulates each core's L1 data-cache as a 2-way associative cache with a block size of 64 Bytes and a total size of $64 \mathrm{~KB}$.

We evaluate different configurations of our LBR/LCR tools. By default, we enable toggling in all tools.

\begin{tabular}{llrllr}
\hline & & & Root & Failure & Log \\
Program & Version & KLOC & Cause & Symptom & Points \\
\hline Sequential-Bug Failures & & & & \\
\hline Apache 1 & 2.0 .43 & 273 & config. & error message & 2534 \\
Apache 2 & 2.2 .3 & 311 & semantic & error message & 2511 \\
Apache 3 & 2.2 .9 & 333 & semantic & error message & 2515 \\
cp & 4.5 .8 & 1.2 & semantic & error message & 108 \\
Cppcheck 1 & 1.58 & 138 & memory & crash & 304 \\
Cppcheck 2 & 1.56 & 131 & memory & crash & 284 \\
Cppcheck 3 & 1.52 & 118 & memory & crash & 225 \\
Lighttpd & 1.4 .16 & 55 & config. & error message & 857 \\
ln & 4.5 .1 & 0.7 & semantic & error message & 29 \\
mv & 6.8 & 4.1 & semantic & error message & 46 \\
paste & 6.10 & 0.5 & memory & hang & 23 \\
PBZIP1 & 1.1 .5 & 5.7 & semantic & error message & 305 \\
PBZIP2 & 1.1 .0 & 4.6 & memory & crash & 269 \\
rm & 4.5 .4 & 1.3 & semantic & error message & 31 \\
sort & 7.2 & 3.6 & memory & crash & 36 \\
Squid1 & $2.5 . S 5$ & 120 & semantic & error message & 2427 \\
Squid2 & 2.3 .54 & 102 & memory & crash & 2096 \\
tac & 6.11 & 0.7 & memory & crash & 21 \\
tar 1 & 1.22 & 82 & semantic & error message & 243 \\
tar 2 & 1.19 & 76 & semantic & error message & 188 \\
\hline Concurrency-Bug Failures & & & & & \\
\hline Apache 4 & 2.0 .50 & 263 & A.V. & crash & 2412 \\
Apache 5 & 2.2 .9 & 333 & A.V. & corrupted log & 2515 \\
Cherokee & 0.98 .0 & 85 & A.V. & corrupted log & 184 \\
FFT & 2.0 & 1.3 & O.V. & wrong output & 59 \\
LU & 2.0 & 1.2 & O.V. & wrong output & 45 \\
Mozilla-JS1 & 1.5 & 107 & A.V. & crash & 343 \\
Mozilla-JS2 & 1.5 & 107 & A.V. & wrong output & 343 \\
Mozilla-JS3 & 1.5 & 107 & A.V. & error message & 343 \\
MySQL1 & 4.0 .18 & 658 & A.V. & crash & 1585 \\
MySQL2 & 4.0 .12 & 639 & A.V. & wrong output & 1523 \\
PBZIP3 & 0.9 .4 & 2.1 & O.V. & crash & 163 \\
\hline & & & & \\
\hline & & & & & \\
\hline
\end{tabular}

Table 4: Features of real-world failures evaluated.

\section{Experimental Results}

\subsection{LBRLOG evaluation}

Our evaluation aims to answer the following questions:

1. Is the LBR record profiled by LBRLOG useful in resolving control-flow uncertainties? 


\begin{tabular}{lcrl}
\hline Application & Useful br. ratio & \#LogSites & Main Log Fun. \\
\hline Apache & 0.86 & 2515 & ap_log_error \\
cp & 0.77 & 108 & error \\
cppcheck & 0.98 & 304 & reportError \\
lighttpd & 0.84 & 857 & log_error_write \\
ln & 0.81 & 29 & error \\
mv & 0.74 & 46 & error \\
paste & 0.86 & 23 & error \\
pbzip & 0.81 & 305 & fprintf \\
rm & 0.79 & 31 & error \\
sort & 0.91 & 36 & error \\
Squid & 0.88 & 2427 & debug \\
tac & 0.89 & 21 & error \\
tar & 0.84 & 243 & open_fatal \\
\hline
\end{tabular}

Table 5: Resolution of control-flow uncertainties by LBRLOG.

2. Is the LBR record profiled by LBRLOG useful in locating failure root causes?

3. Is the runtime performance of LBRLOG suitable for production-run deployment?

\subsubsection{Resolving uncertain control flows}

For each logging site $l$, a branch record in LBR is considered useful if the taken-ness of this branch cannot be inferred based on the execution of $l$ through static control flow analysis. We compute the ratio of useful branches in LBR record entries collected at every failure-logging site in an application. We refer to this ratio as useful branch ratio. Since it is impractical to design inputs to exercise all the logging sites, we implement an LLVM-based analyzer to calculate this ratio. Specifically, given a logging site, the analyzer explores backwards along all possible paths until each path contains 16 branches that could fill LBR and checks which branches are useful. The useful branch ratio shown in Table 5 is averaged across all logging sites in the application.

As shown in Table 5, the average useful branch ratio ranges from 0.74 to 0.98 for all the 6945 logging points across 13 applications. This shows that LBR provides a generic and useful mechanism to resolve control-flow uncertainties.

\subsubsection{Failure diagnostic capability}

To measure the failure-diagnosis capability of LBRLOG, we compare the branches captured by LBRLOG with the patches. We consider LBRLOG to be very helpful in locating the failure root cause, if the patch mainly changes one of the branches recorded by LBRLOG, denoted as $\checkmark$ in Table 6 . We will refer to this branch changed by the patch as root-cause branch. We consider LBRLOG to be helpful, if the patch mainly changes the computation or usage of a condition variable that is involved in one of the branches recorded by LBRLOG, denoted as $\checkmark^{*}$ in Table 6

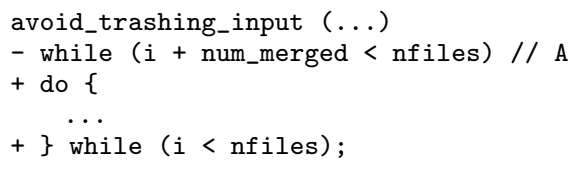

(a) sort patch

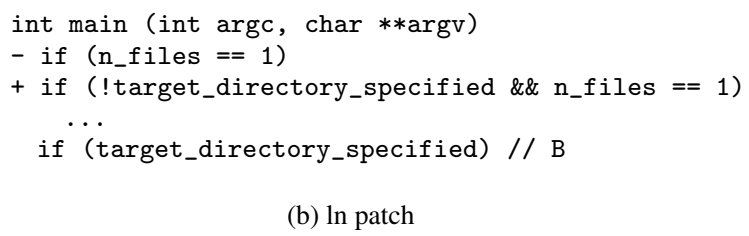

Figure 9: Branches captured by LBRLOG and patches.

As shown in Table 6. LBRLOG is very helpful for diagnosing 16 out of 20 failures. These 16 failures are caused by different types of software bugs: 8 by semantic bugs, 6 by memory bugs, and 2 by configuration errors. As an example, the simplified patch for the sort bug from Section 3.1 is shown in Figure 9a The root cause branch $A$ is recorded as the $3^{\text {rd }}$ latest entry in LBR collected by LBRLOG.

LBRLOG fails to contain the root-cause branch, but is still helpful in diagnosing the remaining 4 failures. For example, the $\ln$ bug has a long error propagation distance. The root-cause branch would have been captured, if LBR had 4 more entries. LBRLOG captures the branch $B$ that is related to the root cause as shown in Figure $9 \mathrm{~b}$.

Table 6 also shows that most root-cause branches are located within the top 8 entries in LBR. This result validates the heuristic that most software bugs have short error propagation distances, and indicates that even on machines with smaller LBR, LBRLOG is still very useful.

Finally, we measure the distance between the LBR branches and the patch, comparing it with the distance between the failure site and the patch. In general, the former is much shorter than the latter. The patches are within 5 lines of code from some LBR branches in 14 out of 20 cases, while only 2 failure sites are within 5 lines of code from the patches. For 13 failures, some LBR branches are more than 30 lines of code closer to the patches than the failure sites, and all these branches are useful LBR records that cannot be inferred by static control-flow analysis. This further shows that LBRLOG can help diagnose failures and design patches.

\subsubsection{Performance}

As shown in Table 6, LBRLOG incurs at most $2.28 \%$ runtime overhead for all the benchmarks, which is suitable for production-run deployment.

The overhead mainly comes from toggling around library functions. Without toggling, the overhead is at most $0.23 \%$ across all benchmarks. This performance improvement comes at the expense of diagnosis capability. As shown 


\begin{tabular}{|c|c|c|c|c|c|c|c|c|c|c|c|}
\hline \multirow[t]{2}{*}{ App. } & \multicolumn{4}{|c|}{ Locate Root Cause } & \multicolumn{2}{|c|}{ Patch distance (LoC) } & \multicolumn{5}{|c|}{ Overhead (\%) } \\
\hline & $\begin{array}{l}\text { LBRLOG } \\
\text { w/ tog. }\end{array}$ & $\begin{array}{l}\text { LBRLOG } \\
\text { w/o tog. }\end{array}$ & LBRA & CBI & $\begin{array}{l}\text { failure } \\
\text { site }\end{array}$ & LBR & $\begin{array}{l}\text { LBRLOG } \\
\text { w/ tog. }\end{array}$ & $\begin{array}{l}\text { LBRLOG } \\
\text { w/o tog. }\end{array}$ & $\begin{array}{l}\text { LBRA } \\
\text { reactive }\end{array}$ & $\begin{array}{c}\text { LBRA } \\
\text { proactive }\end{array}$ & CBI \\
\hline Apache1 & $\checkmark 3$ & $\checkmark 3$ & $\checkmark 1$ & $\checkmark 2$ & $\infty$ & 3 & 0.31 & 0.11 & 0.39 & 3.87 & 3.01 \\
\hline Apache2 & $\checkmark 2^{*}$ & $\checkmark 2^{*}$ & $\checkmark 2^{*}$ & - & $\infty$ & 475 & 0.42 & 0.09 & 0.43 & 4.61 & 5.48 \\
\hline Apache3 & $\checkmark 2$ & $\checkmark 2$ & $\checkmark 1$ & $\checkmark 1$ & 1 & 1 & 0.33 & 0.17 & 0.52 & 3.43 & 2.70 \\
\hline $\mathrm{cp}$ & $\checkmark 2$ & - & $\checkmark 1$ & $\checkmark 1$ & 17 & 15 & 1.77 & 0.23 & 2.13 & 3.61 & 25.90 \\
\hline Cppcheck1 & $\checkmark 5^{*}$ & $\checkmark 5^{*}$ & $\checkmark 1^{*}$ & N/A & $\infty$ & $\infty$ & 2.04 & 0.04 & 2.73 & 5.61 & N/A \\
\hline Cppcheck2 & $\checkmark 3$ & $\checkmark 3$ & $\checkmark 1$ & N/A & $\infty$ & 2 & 0.24 & 0.02 & 0.29 & 2.09 & N/A \\
\hline Cppcheck3 & $\checkmark 6$ & $\checkmark 6$ & $\checkmark 1$ & N/A & $\infty$ & 10 & 1.16 & 0.06 & 1.39 & 4.68 & N/A \\
\hline Lighttpd & $\checkmark 4$ & $\checkmark 4$ & $\checkmark 1$ & - & 0 & 1 & 0.65 & 0.11 & 0.73 & 2.33 & 6.34 \\
\hline $\ln$ & $\checkmark 13^{*}$ & - & $\checkmark 1^{*}$ & $\checkmark 1$ & 254 & 33 & 1.88 & 0.18 & 1.95 & 4.69 & 22.48 \\
\hline $\mathrm{mv}$ & $\checkmark 12$ & $\checkmark 14$ & $\checkmark 1$ & $\checkmark 2$ & 309 & 0 & 1.79 & 0.11 & 2.84 & 5.70 & 15.55 \\
\hline paste & $\checkmark 6$ & - & $\checkmark 1$ & $\checkmark 1$ & 35 & 3 & 1.31 & 0.08 & 1.78 & 2.50 & 14.32 \\
\hline PBZIP1 & $\checkmark 4$ & - & $\checkmark 1$ & N/A & 41 & 1 & 0.29 & 0.07 & 0.34 & 5.73 & N/A \\
\hline PBZIP2 & $\checkmark 1$ & $\checkmark 1$ & $\checkmark 1$ & N/A & 12 & 1 & 0.79 & 0.04 & 0.91 & 4.62 & N/A \\
\hline $\mathrm{rm}$ & $\checkmark 5$ & $\checkmark 5$ & $\checkmark 1$ & $\checkmark 2$ & 31 & 0 & 2.28 & 0.21 & 2.38 & 6.29 & 24.77 \\
\hline sort & $\checkmark 3$ & $\checkmark 5$ & $\checkmark 1$ & $\checkmark 1$ & $\infty$ & 4 & 0.44 & 0.19 & 0.74 & 4.16 & 43.45 \\
\hline Squid1 & $\checkmark 2$ & $\checkmark 2$ & $\checkmark 1$ & - & 123 & 2 & 1.26 & 0.05 & 1.45 & 2.79 & 6.29 \\
\hline Squid2 & $\checkmark 10$ & $\checkmark 10$ & $\checkmark 1$ & $\checkmark 1$ & 59 & 1 & 2.19 & 0.03 & 2.42 & 3.62 & 7.49 \\
\hline tac & $\checkmark 3^{*}$ & $\checkmark 3^{*}$ & $\checkmark 1^{*}$ & $\checkmark 3^{*}$ & $\infty$ & $\infty$ & 2.13 & 0.06 & 2.57 & 2.82 & 26.43 \\
\hline $\operatorname{tar} 1$ & $\checkmark 4$ & $\checkmark 4$ & $\checkmark 1$ & $\checkmark 1$ & $\infty$ & 2 & 0.52 & 0.09 & 0.73 & 3.10 & 14.30 \\
\hline $\operatorname{tar} 2$ & $\checkmark 2$ & - & $\checkmark 1$ & $\checkmark 2$ & 24 & 0 & 0.40 & 0.11 & 0.45 & 2.63 & 9.91 \\
\hline
\end{tabular}

Table 6: Results of LBRLOG and LBRA. (The number $n$ after $\checkmark$ indicates the $n$-th latest LBR entry returned by LBRLOG or the $n$-th top predictor identified by LBRA/CBI; *: root-cause branch is missed, but a branch related to the root cause is identified; "-": no branches related to the root cause are identified; N/A: CBI does not work for C++ applications; $\infty$ : in different files.)

in Table 6 , without toggling, LBRLOG will fail to locate any branch that is related to the patch in 5 cases.

Overall, LBRLOG incurs small overheads across a wide range of applications and is suitable for production-run deployment. We can turn off toggling to satisfy even higher performance requirements.

\subsection{LBRA evaluation}

Our evaluation of LBRA targets the following questions:

1. Is LBRA able to automatically locate root-cause branches?

2. Is the performance of LBRA (reactive and proactive schemes) suitable for production-run deployment?

3. Can LBRA complement CBI, the state-of-the-art production-run failure diagnosis system?

Our experiments configure CBI using its default settings: $1 / 100$ sampling rate; 1000 success runs and 1000 failure runs; with only branch predicates enabled. Our experiments for LBRA only use 10 success runs and 10 failure runs.

LBRA successfully and automatically locates all the 16 root-cause branches contained in LBR as the top 1 failure predictors. It identifies root-cause related branches as top predictors for all the 20 failures. In comparison, CBI identifies root-cause branches as top predictors for 11 out of $15 \mathrm{C}$ program failures. CBI fails to report any root-cause related branches in 3 cases, where its random sampling missed the relevant predicates too many times.
The above diagnosis results are achieved with LBRA analyzing much fewer failure runs than CBI (10 vs. 1000). When we applied CBI to 500, instead of 1000, failure-run profiles, CBI failed to identify any useful failure predictors for 10 out of $15 \mathrm{C}$-program failures. This difference would be crucial for software that is not deployed on millions of machines or failures that do not occur very frequently.

As shown in Table 6, the run-time overhead of LBRA (reactive mode) is always less than $3 \%$, well suitable for production-run deployment. The overhead of LBRA in proactive mode is slightly larger, ranging between $2.09 \%$ and $6.29 \%$. It is a good choice for software where updates are infrequent or very expensive. CBI incurs an average overhead of $15.23 \%$, much larger than LBRA, mainly due to the instrumentation done by CBI to performs sampling.

The results show that LBRA well complements CBI.

\subsection{LCR evaluation}

Our evaluation tries to answer the following questions:

1. Can LCRLOG help diagnose concurrency-bug failures?

2. Can LCRA automatically locate the root causes of concurrency-bug failures?

3. Can LCR complement PBI and CCI, the state-of-the-art production-run concurrency-bug failure diagnosis tools?

LCRLOG We consider LCRLOG to directly locate the failure root cause, if the LCR profiled by it contains the failure-predicting coherence event (defined in Section 4.2.2). 
As shown in Table 7, LCRLOG directly locates the root cause for 7 out of 11 concurrency-bug failures, which cover different types of root causes and symptoms.

LCRLOG does not directly locate the root cause for Apache5, Cherokee, and Mozilla-JS2 failures, because these bugs cause silent data corruption with no failure logging near the root cause. The MySQL1 failure is caused by a WRW atomicity violation. As shown in Table 3 , since the failurepredicting event does not exist in the failure thread, it is not profiled by LCRLOG.

Further, as shown in Table 7 the capacity of LCR is not a problem for the failures we evaluated. Using the more space-saving configuration, the failure-predicting events are always contained in top $4 \mathrm{LCR}$ entries. Even with the more space-consuming configuration, the failure-predicting events are still located within top 12 LCR entries.

\begin{tabular}{lccc}
\hline ID & LCRLOG (Conf1) & LCRLOG (Conf2) & LCRA \\
\hline Apache4 & $\checkmark 3$ & $\checkmark 5$ & $\checkmark 1$ \\
Apache5 & - & - & - \\
Cherokee & - & - & - \\
FFT & $\checkmark 4$ & $\checkmark 6$ & $\checkmark 1$ \\
LU & $\checkmark 4$ & $\checkmark 6$ & $\checkmark 1$ \\
Mozilla-JS1 & $\checkmark 3$ & $\checkmark 8$ & $\checkmark 1$ \\
Mozilla-JS2 & - & - & - \\
Mozilla-JS3 & $\checkmark 3$ & $\checkmark 11$ & $\checkmark 1$ \\
MySQL1 & - & - & - \\
MySQL2 & $\checkmark 3$ & $\checkmark 9$ & $\checkmark 1$ \\
PBZIP3 & $\checkmark 3$ & $\checkmark 7$ & $\checkmark 1$ \\
\hline
\end{tabular}

Table 7: Failure diagnosis capability of LCR. (A number $n$ after the $\checkmark$ indicates the $n$-th latest entry returned by LCRLOG or the $n$-th best failure predictor returned by LCRA is the root-cause failure-predicting event; Conf1 is the spacesaving configuration of LCR; Conf2 is the space-consuming configuration of LCR; LCRA uses Conf2.)

LCRA We evaluate whether LCRA can automatically locate the failure-predicting event by applying LCRA to 10 failure runs and 10 success runs in each case.

LCRA successfully ranks the failure-predicting event at the top for all the 7 failures where the failure-predicting event is captured by LCRLOG. For example, for the failure discussed in Section 3.2 (Mozilla-JS3), LCRA automatically locates the invalid state observed by $a_{2}$ as the top failure predictor.

We expect LCRA to well complement PBI and CCI. In terms of performance, CCI incurs up to 10 times slow down, due to its software based sampling schemes. We expect LCRA to have similar performance as LBRA, which would be comparable to or slightly better than PBI. In terms of failure-diagnosis capability, LCRA is slightly worse than PBI, which can successfully diagnose all the 11 failures, and comparable with CCI, which can successfully diagnose 7 out of the 11 failures.
The biggest advantage of LCRA is its short failurediagnosis latency. LCRA achieves the above diagnosis results using only 10 failure-run profiles, while PBI and CCI need the failures to occur hundreds to thousands of times [2, 18]. This is especially a problem for concurrency-bug failures that often occur non-deterministically and rarely.

\section{Related Work}

We briefly discuss related work that has not been discussed.

Hardware performance monitoring unit The branch tracing facility has been used in several recent work, but has never been used for production-run failure diagnosis. THeME uses LBR for testing coverage analysis [40]. Recent work conducts vulnerability or malware analysis on branch traces generated by the branch tracing facility [41, 46]. Intel GNU* GDB tool [16] uses BTS to store all executed branches in an OS-provided ring buffer.

Our work uses the branch tracing facilities for different purposes from previous work, which leads to different designs. For example, all the above work collects the branch trace of the whole execution, while we focus on the LBR collected at the failure site. Our system aims to achieve very small run-time overhead for production-run deployment, while the above work does not share the same goal. Some of them [16, 46] intentionally use BTS which has higher overhead. THeME [40] uses LBR, but still incurs much larger overhead than our tools. The reason is that THeME's design goal, computing testing coverage, demands periodic LBR profiling throughout program execution. Instead, LBRLOG only profiles LBR when software fails.

General hardware performance counters have been used to identify malware [10] and detect data races [11,37]. These tools all monitor and analyze the whole execution, instead of focusing on the execution leading to a failure. Race detectors [11, 37] focus on one specific type of software bugs, and cannot help diagnose general software failures. In addition, not being guided by a specific failure, race detectors would report a large number of false positives [18].

Production-run failure diagnosis Record-and-replay techniques [1, 13, 19, 20] can help diagnose production-run failures. However, they could hurt the end users' privacy and incur large overhead for deterministic replay of multithreaded software. Triage [38] diagnoses production-run failures by applying automated bug detection during on-site replay, which is supported by OS modifications. Overall, record-and-replay techniques and our system can complement each other in failure diagnosis.

An adaptive version of CBI was proposed based on dynamic binary rewriting [4]. CBI-adaptive iteratively changes sampling locations based on the failure location and the diagnosis results from earlier iterations. Without knowing the exact control-flow leading to failures, CBI-adaptive needs hundreds of iterations and evaluates about $40 \%$ of all program predicates before it finishes failure diagnosis. 
Hardware support for bug detection A lot of work has been done to speed up sequential-bug detection through hardware support [39, 49]. Different from LBRA and LBRLOG, most of these proposals rely on non-existing hardware.

A lot of work has proposed detecting concurrency bugs through hardware support [6, 7, 24, 26, 32, 33, 42, 50]. LCR has drawn inspiration from these work. However, since previous work focuses on bug detection, it requires the hardware and software system to contiguously monitor and analyze program execution, while maintaining a long execution history. Many bug detectors need to report suspicious execution patterns even if they do no lead to failures. LCR leverages the unique need of failure diagnosis and designs a very simple hardware extension to maintain a short-term execution history.

Bugaboo [26] detects a wide variety of concurrency bugs by identifying rare communication patterns. The communication graph in Bugaboo associates with every memory instruction $m$ from thread $t$ a context, the sequence of communication events observed by $t$ immediately prior to $m$. A LCR record is similar to a context, as they both contain a short-term history of thread interaction. However, Bugaboo and our system have very different designs, because they have different goals - Bugaboo detects concurrency bugs even without failure information; our system helps diagnose production-run failures. Bugaboo maintains and checks the context of every memory instruction in every thread throughout the execution. Our system leverages the unique need of failure diagnosis and only uses LCR collected in the failure thread right before the failure. In addition, Bugaboo extends existing cache-coherence protocol to collect context events, while each LCR event is already supported by existing hardware performance monitoring unit.

ECMon [28] proposes a hardware extension that allows custom handlers to execute whenever certain type of cache (coherence) events happen. ECMon and LCR aim for different usage scenarios, and hence have different designs. Software uses ECMon to process cache events throughout the program execution; our system uses LCR to access the last few cache coherence events at the moment of failure. Also different from ECMon, the design of LCR is built upon existing hardware performance monitoring unit.

\section{Conclusion}

We design and implement a novel mechanism that leverages hardware's short-term memory to support productionrun failure diagnosis. We identify an existing hardware performance monitoring unit, LBR, and design a simple hardware extension, LCR, to maintain a short-term memory of hardware events that are useful for failure diagnosis. Our evaluation of 31 sequential-bug and concurrency-bug failures from 18 open-source software shows that our LBR/LCR based tools can effectively enhance failure logging and automatically locate failure root causes with small run-time overhead. We believe that our LBR/LCR system provides a good balance between run-time performance, diagnosis latency, and diagnosis capability. Our experience demonstrates that short-term memory is sufficient for diagnosing a wide variety of real-world failures. It also shows that a very simple hardware-extension can provide significant help for production-run failure diagnosis.

\section{Acknowledgments}

We thank the anonymous reviewers for their insightful feedback which has substantially improved the content and presentation of this paper; Ding Yuan and the Opera Group for their tremendous help in sharing the benchmarks; Ben Liblit, Piramanayagam Nainar and Peter Ohmann for their valuable guidance on using the CBI framework; Jason Mars and Asim Kadav for their insightful comments on designing the kernel interface to LBR. This work is supported in part by NSF grants CCF-1018180, CCF-1054616, and CCF1217582; and a Clare Boothe Luce faculty fellowship.

\section{References}

[1] G. Altekar and I. Stoica. ODR: Output-deterministic replay for multicore debugging. In SOSP, 2009.

[2] J. Arulraj, P.-C. Chang, G. Jin, and S. Lu. Production-run software failure diagnosis via hardware performance counters. In ASPLOS, 2013.

[3] P. Arumuga Nainar. Applications of Static Analysis and Program Structure in Statistical Debugging. PhD thesis, University of Wisconsin - Madison, 2012.

[4] P. Arumuga Nainar and B. Liblit. Adaptive bug isolation. In ICSE, 2010.

[5] B. Buck and J. K. Hollingsworth. An API for runtime code patching. Int. J. High Perform. Comput. Appl., 2000.

[6] L. Ceze, P. Montesinos, C. von Praun, and J. Torrellas. Colorama: Architectural support for data-centric synchronization. In $H P C A, 2007$.

[7] L. Chew and D. Lie. Kivati: Fast detection and prevention of atomicity violations. In EuroSys, 2010.

[8] CNNMoneyTech. Is knight's $\$ 440$ million glitch the costliest computer bug ever? http://money.cnn.com/2012/08/09/ technology/knight-expensive-computer-bug/index.html

[9] J. Demme and S. Sethumadhavan. Rapid identification of architectural bottlenecks via precise event counting. In ISCA, 2011.

[10] J. Demme, M. Maycock, J. Schmitz, A. Tang, A. Waksman, S. Sethumadhavan, and S. Stolfo. On the feasibility of online malware detection with performance counters. In ISCA, 2013.

[11] J. L. Greathouse, Z. Ma, M. I. Frank, R. Peri, and T. M. Austin. Demand-driven software race detection using hardware performance counters. In ISCA, 2011.

[12] W. Gu, Z. Kalbarczyk, R. K. Iyer, and Z. Yang. Characterization of Linux kernel behavior under errors. In DSN, 2003. 
[13] D. R. Hower, P. Montesinos, L. Ceze, M. D. Hill, and J. Torrellas. Two hardware-based approaches for deterministic multiprocessor replay. Commun. ACM, 52(6), June 2009.

[14] Intel. Nehalem PMU programming guide. http://software. intel.com/sites/default/files/76/87/30320 2010.

[15] Intel. Intel 64 and IA-32 architectures software developers manual. http://download.intel.com/products/processor/ manual/325462.pdf 2013.

[16] Intel. GDB - the GNU* project debugger for Intel architecture. http://software.intel.com/en-us/articles/ intel-system-studio-gdb\#Trace. March 2013.

[17] J. L. Lions et. al. ARIANE 5 Flight 501 Failure - report by the inquiry board. http://sunnyday. mit.edu/accidents/ Ariane5accidentreport.html.

[18] G. Jin, A. Thakur, B. Liblit, and S. Lu. Instrumentation and sampling strategies for cooperative concurrency bug isolation. In OOPSLA, 2010.

[19] S. T. King, G. W. Dunlap, and P. M. Chen. Operating systems with time-traveling virtual machines. In Usenix Annual Technical Conference, 2005.

[20] D. Lee, B. Wester, K. Veeraraghavan, S. Narayanasamy, P. M. Chen, and J. Flinn. Respec: Efficient online multiprocessor replay via speculation and external determinism. In ASPLOS, 2010.

[21] N. Leveson and C. S. Turner. An investigation of the Therac25 accidents. In IEEE Computer, 1993.

[22] B. Liblit, A. Aiken, A. X. Zheng, and M. I. Jordan. Bug isolation via remote program sampling. In PLDI, 2003.

[23] B. Liblit, M. Naik, A. X. Zheng, A. Aiken, and M. I. Jordan. Scalable statistical bug isolation. In PLDI, 2005.

[24] S. Lu, J. Tucek, F. Qin, and Y. Zhou. AVIO: Detecting atomicity violations via access interleaving invariants. In ASPLOS, 2006.

[25] S. Lu, S. Park, E. Seo, and Y. Zhou. Learning from mistakes - a comprehensive study of real world concurrency bug characteristics. In ASPLOS, 2008.

[26] B. Lucia and L. Ceze. Finding concurrency bugs with contextaware communication graphs. In MICRO, 2009.

[27] C.-K. Luk, R. Cohn, R. Muth, H. Patil, A. Klauser, G. Lowney, S. Wallace, V. J. Reddi, and K. Hazelwood. Pin: Building customized program analysis tools with dynamic instrumentation. In PLDI, 2005.

[28] V. Nagarajan and R. Gupta. ECMon: Exposing cache events for monitoring. In ISCA, 2009.

[29] S. Narayanasamy, G. Pokam, and B. Calder. BugNet: Continuously recording program execution for deterministic replay debugging. In ISCA, 2005.

[30] PCWorld. Nasdaq's Facebook Glitch Came From Race Conditions. http://www.pcworld.com/businesscenter/ article/255911/nasdaqs_facebook_glitch_came_from_race_ conditions.html

[31] C. Pedersen and J. Acampora. Intel code execution trace resources. http://noggin.intel.com/content/ intel-code-execution-trace-resources 2012.
[32] M. Prvulovic. CORD: Cost-effective (and nearly overheadfree) order-reordering and data race detection. In $H P C A$, 2006.

[33] M. Prvulovic and J. Torrellas. ReEnact: Using thread-level speculation mechanisms to debug data races in multithreaded codes. In ISCA, 2003.

[34] F. Qin, J. Tucek, J. Sundaresan, and Y. Zhou. Rx: Treating bugs as allergies c a safe method to survive software failures. In SOSP, 2005.

[35] R. Santelices, J. A. Jones, Y. Yu, and M. J. Harrold. Lightweight fault-localization using multiple coverage types. In ICSE, 2009.

[36] SecurityFocus. Software bug contributed to blackout. http: //www.securityfocus.com/news/8016

[37] T. Sheng, N. Vachharajani, S. Eranian, R. Hundt, W. Chen, and W. Zheng. RACEZ: A lightweight and non-invasive race detection tool for production applications. In ICSE, 2011.

[38] J. Tucek, S. Lu, C. Huang, S. Xanthos, and Y. Zhou. Triage: Diagnosing production run failures at the user's site. In SOSP, 2007.

[39] G. Venkataramani, B. Roemer, Y. Solihin, and M. Prvulovic. MemTracker: Efficient and programmable support for memory access monitoring and debugging. In HPCA, 2007.

[40] K. Walcott-Justice, J. Mars, and M. L. Soffa. THeME: A system for testing by hardware monitoring events. In ISSTA, 2012.

[41] C. Willems, R. Hund, A. Fobian, D. Felsch, T. Holz, and A. Vasudevan. Down to the bare metal: Using processor features for binary analysis. In ACSAC, 2012.

[42] J. Yu and S. Narayanasamy. A case for an interleaving constrained shared-memory multi-processor. In ISCA, 2009.

[43] D. Yuan, H. Mai, W. Xiong, L. Tan, Y. Zhou, and S. Pasupathy. SherLog: Error diagnosis by connecting clues from run-time logs. In ASPLOS, 2010.

[44] D. Yuan, J. Zheng, S. Park, Y. Zhou, and S. Savage. Improving software diagnosability via log enhancement. In ASPLOS, 2011.

[45] D. Yuan, S. Park, P. Huang, Y. Liu, M. M. Lee, Y. Zhou, and S. Savage. Be conservative: Enhancing failure diagnosis with proactive logging. In OSDI, 2012.

[46] L. Yuan, W. Xing, H. Chen, and B. Zang. Security breaches as PMU deviation: Detecting and identifying security attacks using performance counters. In APSys, 2011.

[47] W. Zhang, C. Sun, and S. Lu. ConMem: Detecting severe concurrency bugs through an effect-oriented approach. In ASPLOS, 2010.

[48] W. Zhang, J. Lim, R. Olichandran, J. Scherpelz, G. Jin, S. Lu, and T. Reps. ConSeq: Detecting concurrency bugs through sequential errors. In ASPLOS, 2011.

[49] P. Zhou, F. Qin, W. Liu, Y. Zhou, and J. Torrellas. iWatcher: Efficient architecture support for software debugging. In ISCA, 2004.

[50] P. Zhou, R. Teodorescu, and Y. Zhou. Hard: Hardwareassisted lockset-based race detection. In HPCA, 2007. 